\title{
ISBER's Role in Shaping Global Policies and Regulations
}

\author{
Marianna J. Bledsoe ${ }^{1-3}$ and Debra Leiolani Garcia ${ }^{4,5}$
}

$\mathrm{O}_{\mathrm{B}}^{\mathrm{N}}$ NE OF THE major goals of the International Society for Biological and Environmental Repositories (ISBER www.isber.org) is to serve as the voice for repositories to influence regulations and policies affecting biobanking worldwide. The regulatory and policy landscape related to biobanking is rapidly evolving, particularly in the areas of privacy, data protections, and human subjects research. These developments can have a dramatic impact on biobanking operations and biospecimen and data sharing. ISBER, through its Science Policy (SciPol) Advisory Committee, helps to shape important global and regional regulations and policies related to biorepositories in a variety of ways. The goal is to facilitate biobanking and biospecimen and data research while ensuring the ethical conduct of such research. An additional broader goal is to promote a deeper understanding of the laws and regulations governing therapeutic innovation and the protection of human research participants on the global stage, particularly those related to biobanking.

\section{Comments on Draft Regulations and Policy Developments}

The ISBER SciPol Committee (https://www.isber.org/ page/Committees) monitors emerging policy and regulatory developments worldwide, analyzes their impact on biobanking and biospecimen research, and provides public input on them. It encourages and facilitates open and collaborative discussion of nonproprietary issues in research and development, with a specific focus on biobanking and biospecimen research.

Policy statements submitted by ISBER include those in response to requests for comments on important guidelines related to biobanking, including the WMA Declaration of Taipei on Ethical Considerations Regarding Health Databases and Biobanks, ${ }^{1}$ the Council of Europe Recommendations on Research on Biological Materials of Human Origin, ${ }^{2}$ and the CIOMS International Ethical Guidelines for Biomedical Research Involving Human Subjects. ${ }^{3}$ In addition, comments were submitted on important regional regulations such as the revisions to the U.S. Common Rule, ${ }^{4}$ the Euro- pean Union General Data Protection Regulations (GDPR), ${ }^{5}$ the Proposed Changes to TCPS 2 (2014) Guidance for Research involving human cells and cell lines, ${ }^{6}$ and the Australian National Statement on Ethical Conduct in Human Research. ${ }^{7}$ Of note, ISBER comments were cited in the U.S. Presidential Commission for the Study of Bioethical Issues report entitled, "Anticipate and Communicate: Ethical Management of Incidental and Secondary Findings in the Clinical, Research, and Direct-to-Consumer Contexts." 8 A complete list of policy statements with copies is available for download at (https://www.isber.org/page/PositionStatements).

\section{Participation in the Research, Development, and Regulatory Roundtable}

Another mechanism by which ISBER is helping to shape regulations and policies globally is by participating in the Research, Development, and Regulatory Roundtable (R3) (https://mrctcenter.org/blog/projects/research-developmentand-regulatory-roundtable-r3-of-the-mrct-center-and-ropesgray-llp/). The R3 Regulatory Roundtable is an impartial forum wherein policy makers, legal counsel, academicians, industry representatives, and global regulators present, discuss, and deliberate cutting-edge issues in drug and device development, human subjects research, and regulatory oversight of clinical trials. The R3 Roundtable strives to foster a broader understanding of the laws and regulations that influence therapeutic innovation, product approval, and the protection of human research participants on the global stage by gathering stakeholders from academia, industry, and regulatory agencies in a nonpartisan environment. The R3 is jointly organized and coordinated by the Multi-Regional Clinical Trials Center of Brigham and Women's Hospital and Harvard, along with Ropes \& Gray LLP. ISBER is currently one of several sponsoring organizations.

One of the major issues that R3 has been exploring is the impact of the EU GDPR ${ }^{5}$ on clinical research and global data privacy, including its effect on biobanking. ISBER was represented at an important meeting organized by members of R3 held in Brussels in November 2019 regarding the impact

${ }^{1}$ Independent Consultant, Colorado Springs, Colorado, USA.

${ }^{2}$ Chair, ISBER Science Policy Committee.

${ }^{3}$ Deputy Editor, Biopreservation and Biobanking.

${ }^{4}$ Independent Consultant, San Francisco, California, USA.

${ }^{5}$ ISBER Past President, 2020-2021. 
of the GDPR on clinical research. The meeting provided an opportunity for a dialogue with policy officials from the European Commission. The meeting formed the basis for the development of a consensus document about the challenges of the GDPR for clinical research and what is needed to overcome them that was subsequently submitted to the European Commission. This article helped to inform ISBER's Comments on the application of the GDPR to biomedical research (https://cdn.ymaws.com/www.isber.org/resource/resmgr/ docs/ISBER_Comments_on_Applicatio.pdf). Other topics relevant to biobanking that R3 has been exploring include genetic data sharing, obligations of informed consent (and broad consent) under the Revised Common Rule, regulation and use in clinical research of in vitro diagnostic devices in the United States, and the impact of the COVID-19 pandemic on multinational projects and collaborations.

The R3 has also reviewed updates to China's Human Genetic Resources (HGR) regulation and discussed how the changes will alter sharing biospecimens and biospecimen data with China. The HGR updates were contextualized within China's broader evolving legislation that aims to protect national security by protecting data.

Participation in R3 has benefited ISBER and the biobanking community in a variety of ways. It has provided ISBER an opportunity to more fully engage big pharma in the activities of our society. R3 participants are based at sites worldwide allowing R3 to address global regulatory and policy issues. In addition, participation in R3 allows ISBER to expand its global presence.

R3 has been recognized as one of the leading voices in the field of policy and regulatory issues related to international research and biobanking. Members of R3 have published numerous documents 9,10 explaining the various provisions of the GDPR, providing useful resources for the biobanking community, and recommendations for policy makers. These recommendations, if adopted by policy makers, will facilitate research involving biospecimen and data sharing globally and, therefore, likely to significantly benefit ISBER members as a result.

ISBER's participation in forums such as R3 provides benefits that are very important in positioning ISBER as a major policy leader in the field of biobanking and regulatory issues regarding human subjects, data protection, and data sharing. R3 has provided access to information and expertise that ISBER would not ordinarily have and has helped shape important policy developments globally related to biobanking.

\section{Engagement with ISBER Membership to Monitor and Shape Regional Policy}

The ISBER SciPol Committee routinely monitors the global regulatory and policy landscape to identify emerging issues that may impact the biobanking community. However, engagement of the broader ISBER membership is necessary to ensure that regional policy issues are addressed. Specific regional policy developments and issues need to be brought to ISBER's attention so that ISBER can share information about them and contribute with policy statements as appropriate. A plan has been developed to support regional policy engagement that includes soliciting input on the ISBER Group Forum, in ISBER Corner articles, and other media on a regular basis, as well as through the ISBER Regional Ambassadors (https://www.isber.org/page/MeetTheAmbassadors). In addition, regional policy specialists will be identified to help monitor emerging regional policy issues and advise the society about opportunities to provide public comments. Such engagement is important for ISBER to serve as a true global voice in the area of regulations and policies, supporting where possible the ethical conduct of biobanking and biospecimen research, global harmonization, and cross border sharing of biospecimens and data.

\section{Conclusion}

In conclusion, the ethical, legal, and regulatory landscape related to biobanking continues to evolve along with new scientific developments and the evolution in ethical thinking. ISBER, through the ISBER SciPol Committee, will continue to monitor developments in this area, provide input when needed, and engage with our collaborators and other stakeholders to facilitate biobanking and biospecimen research while promoting the ethical conduct of such research.

\section{Acknowledgments}

The authors acknowledge the important contributions of the ISBER Science Policy Advisory Committee in reviewing draft regulations and policies, analyzing them and preparing public policy statements on behalf of ISBER, as well as the critical work of the members of R3.

\section{References}

1. Council of Europe. Recommendations on research on biological materials of human origin. 2016. Available from: https://search.coe.int/cm/Pages/result_details.aspx?ObjectId= 090000168064e8ff (accessed December 21, 2020).

2. World Medical Association. WMA declaration of Taipei on ethical considerations regarding health databases and biobanks. 2016. Available from: www.wma.net/policies-post/ wma-declaration-of-taipei-on-ethical-considerations-regardinghealth-databases-and-biobanks/ (accessed December 21, 2020).

3. Council for International Organizations of Medical Sciences. International ethical guidelines for health-related research involving humans. Council of Europe. Recommendations on Research on Biological Materials of Human Origin. 2016. Available from: https://search.coe.int/cm/Pages/result_details .aspx ? ObjectId=090000168064e8ff (accessed December 21, 2020).

4. Department of Health and Human Services, Office of Human Research Protections, The Revised Common Rule. Available from: https:/www.hhs.gov/ohrp/regulations-andpolicy/regulations/finalized-revisions-common-rule (accessed December 21, 2020).

5. European Commission. Data Protection in the EU. Available from: https:/ec.europa.eu/info/law/law-topic/data-protection/ data-protection-eu_en (accessed December 21, 2020).

6. Government of Canada. Panel on Research Ethics. Proposed Changes to TCPS 2 (2014) Guidance for Research involving Human Cells and Cell Lines. Available from: https://ethics.gc.ca/eng/nr-cp_2017-09-29.html (accessed December 21, 2020).

7. NHMRC. National statement on ethical conduct in human research. 2018. Available from: https://www.nhmrc.gov.au/ about-us/publications/national-statement-ethical-conducthuman-research-2007-updated-2018 (accessed on December $21,2020)$. 
8. US Presidential Commission for the Study of Bioethical Issues. Anticipate and communicate: ethical management of incidental and secondary findings in the clinical, research, and direct-to-consumer contexts. 2013. Available from: https://www.genome.gov/Pages/PolicyEthics/Health Issues/Anticipate_Communicate.pdf (accessed on December 21, 2020).

9. Peloquin D, DiMaio M, Bierer B, Barnes M. Disruptive and avoidable: GDPR challenges to secondary research uses of data. Eur J Hum Genet 2020;28:697-705.

10. Bovenberg J, Peloquin D, Bierer B, Barnes M, Knoppers, BM. How to fix the GDPR's frustration of global biomedical research. Science 2020;370:40-42.
Address correspondence to:

Marianna J. Bledsoe, MA

Colorado Springs, CO 80921

USA

E-mail: mariannabledsoe@gmail.com

Debra Leiolani Garcia, MPA San Mateo, CA 94401 USA

E-mail: dleiolani@gmail.com 